\title{
LINC00963 silencing inhibits the proliferation and migration of high glucose-induced retinal endothelial cells via targeting $\mathrm{miR}-27 \mathrm{~b}$
}

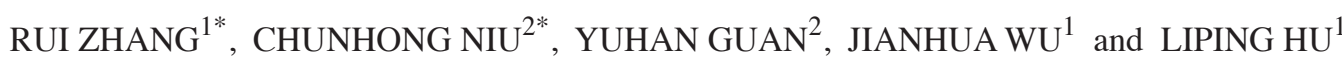 \\ ${ }^{1}$ Fundus Disease Department, Aier Eye Hospital of Wuhan University, Wuhan, Hubei 430063; \\ ${ }^{2}$ Department of Nursing, The Tianjin 4th Central Hospital, Tianjin 300140, P.R. China
}

Received October 30, 2020; Accepted March 18, 2021

DOI: $10.3892 /$ etm.2021.10709

\begin{abstract}
The association between long intergenic non-protein-coding RNA 963 (LINC00963) and diabetes has not been fully elucidated. Therefore, the present study aimed to investigate the effect of the long non-coding RNA LINC00963 on diabetic retinopathy (DR), in order to provide a new therapeutic target for this condition. Human retinal capillary endothelial cells (HRECs) were induced with high concentrations of glucose to establish a DR model. The expression levels of LINC00963, cell viability, the protein expression levels of proliferating cell nuclear antigen (PCNA) and Ki67, and the migratory capacity of HRECs were determined using reverse transcription-quantitative PCR (RT-qPCR), Cell Counting Kit-8 assay, western blot analysis, and wound healing and Transwell assays, respectively. Furthermore, the Encyclopedia of RNA Interactomes database was used to predict the binding targets of LINC00963, and luciferase reporter assay was used to verify the direct binding of microRNA (miR)-27b to LINC00963. RT-qPCR was also utilized to measure the expression levels of miR-27b, PCNA and Ki67. The results demonstrated that LINC00963 silencing inhibited glucose-induced HREC proliferation and migration, and downregulated PCNA and Ki67 expression. Following transfection with miR-27b inhibitor, cell proliferation and migration were notably enhanced, and the protein expression levels of PCNA and Ki67 were increased. Taken together, the results of the present study suggested that the LINC00963/miR-27b axis may regulate the proliferation and
\end{abstract}

Correspondence to: Dr Liping Hu, Fundus Disease Department, Aier Eye Hospital of Wuhan University, 481 Zhongshan Road, Wuchang, Wuhan, Hubei 430063, P.R. China

E-mail: hulipinghlpp@163.com

*Contributed equally

Key words: diabetic retinopathy, long intergenic non-protein-coding RNA 963, proliferation, migration migration of glucose-induced HRECs. Therefore, LINC00963 may be considered as a potential therapeutic target for DR.

\section{Introduction}

Diabetes is a metabolic syndrome characterized by elevated blood glucose levels and is caused by insufficient insulin production and/or peripheral tissue resistance to the action of insulin (1).

The ninth edition of the Diabetes Overview, published by the International Diabetes Federation (IDF), has estimated that $>500$ million adults worldwide suffer from diabetes mellitus, with $\sim 1.2$ billion adults with diabetes in China alone (2). Diabetic retinopathy (DR) is the most common microvascular complication and a major cause of blindness in patients with diabetes (3). It has been reported that DR is closely associated with damage to the retinal microvascular system mediated by a high-glucose environment. DR can affect the structure of the retina, leading to its impaired metabolism and dysfunction (4).

Long non-coding RNAs (lncRNAs) are a class of non-coding RNA molecules that are $>200$ nucleotides in length. IncRNAs can exert their biological functions through several pathways (5). MicroRNAs (miRNAs/miRs) are small non-coding single-stranded RNAs, 18-25 nucleotides in length, which act mainly through complementary pairing with downstream target mRNAs to promote their degradation or suppression $(6,7)$. miRNAs are involved in several physiological processes, including cell proliferation, apoptosis, signal transduction, differentiation, metabolism and hormone secretion, as well as in the maintenance of the stemness potential of embryonic stem cells. In addition, miRNAs regulate human growth and development, and allow the body to adapt to its environment (8). However, the role of miRNAs in DR has not been extensively investigated. miR-27b is commonly dysregulated in human cancers, such as glioma, cervical and breast cancer, and is downregulated in lung adenocarcinoma, gastric cancer, acute myeloid leukemia, colorectal, prostate and bladder cancer (9).

The aim of the present study was to investigate the effect of the long intergenic non-protein-coding RNA 963 (LINC00963) on the proliferation and migration of high glucose-induced human retinal capillary endothelial cells (HRECs). 


\section{Materials and methods}

Cell culture and establishment of the DR cell model. HRECs were purchased from the China Infrastructure of Cell Line Resources, Institute of Basic Medical Sciences, Chinese Academy of Medical Sciences. Cells were maintained in endothelial cell medium (ScienCell Research Laboratories, Inc.) supplemented with 5\% FBS (Beijing Solarbio Science \& Technology Co., Ltd.), $100 \mu \mathrm{g} / \mathrm{ml}$ penicillin and $100 \mu \mathrm{g} / \mathrm{ml}$ streptomycin (Gibco; Thermo Fisher Scientific, Inc.), at $37^{\circ} \mathrm{C}$ in a humidified atmosphere of $5 \% \mathrm{CO}_{2}$. The cells were divided into the following groups: i) Normal glucose (NG) group (5 mM normal glucose); ii) high glucose (HG) group (30 mM glucose); and iii) mannitol (MA) group (24.5 mmol/1 mannitol + $5.5 \mathrm{mmol} / 1$ glucose).

Cell viability. Cell viability was assessed using a Cell Counting Kit-8 (CCK-8) assay (Dojindo Molecular Technologies, Inc.), according to the manufacturer's instructions. Briefly, HRECs were resuspended in endothelial cell medium (ScienCell Research Laboratories, Inc.) and seeded into 96-well plates at a density of 5,000 cells/well. Subsequently, a total of $10 \mu 1$ CCK-8 solution was added to each well and cells were incubated for $1 \mathrm{~h}$ at room temperature. Finally, the optical density in each well was measured at a wavelength of $450 \mathrm{~nm}$ to determine cell viability.

Prediction of target genes. The Encyclopedia of RNA Interactomes (ENCORI; starBase v2.0 https://starbase. sysu.edu.cn) is an open-source platform for evaluating the miRNA-ncRNA, miRNA-mRNA, ncRNA-RNA, RNA-RNA, RBP-ncRNA and RBP-mRNA interactions from CLIP-seq, degradome-seq and RNA-RNA interactome data.

Dual-luciferase reporter assay. The sequences of wild-type (WT) LINC00963 (accession no. NC_000009; 5'-GUG GGGCUGUGUUGACUGUGAG-3') and mutant (MUT) LINC00963 (5'-GUGGGGCUGUGUUGUGACACUU-3') were amplified by Shanghai GenePharma Co., Ltd., cloned into a luciferase reporter vector pGL3 (cat. no. E1761; Promega Corporation), named WT LINC00963-Luc and MUT LINC00963-Luc respectively. Cell were co-transfected with miR-27b mimic (50 nM, 5'-UUCACAGUGGCUAAG UUCUGC-3'; Shanghai GenePharma Co., Ltd.) or miR-NC (50 Nm, 5'-UAACACAACUCUAGAGUAGGA-3'; Shanghai GenePharma Co., Ltd.) and WT or MUT LINC00963-Luc into HRECs using the Lipofectamine ${ }^{\circledR} 3000$ transfection reagent (Invitrogen; Thermo Fisher Scientific, Inc.) with $37^{\circ} \mathrm{C}$ for $48 \mathrm{~h}$. The Dual-Luciferase ${ }^{\circledR}$ Reporter Assay System (cat. no. E1910, Promega Corporation) was used to evaluate the relative luciferase signals. The relative luciferase activity was measured at a wavelength of $410 \mathrm{~nm}$ using a plate reader (BD Biosciences) and normalized to Renilla luciferase activity.

Reverse transcription-quantitative PCR (RT-qPCR). Total RNA was extracted from HRECs using TRIzol ${ }^{\circledR}$ reagent (Invitrogen; Thermo Fisher Scientific, Inc.) and cDNA was synthesized using the PrimeScript RT kit (Takara Bio, Inc.) at $37^{\circ} \mathrm{C}$ for $15 \mathrm{~min}$ and at $85^{\circ} \mathrm{C}$ for $5 \mathrm{sec}$. The cDNA solution was then stored at $-80^{\circ} \mathrm{C}$. Subsequently, qPCR was performed using the iTaq ${ }^{\mathrm{TM}}$ Universal SYBR-Green Supermix (Bio-Rad Laboratories, Inc.) on an ABI 7500 Real-Time qPCR system (Applied Biosystems; Thermo Fisher Scientific, Inc.) using the following conditions: Pre-denaturation at $95^{\circ} \mathrm{C}$ for $3 \mathrm{~min}$, followed by 40 cycles of denaturation $95^{\circ} \mathrm{C}$ for $30 \mathrm{sec}$, annealing at $58^{\circ} \mathrm{C}$ for $30 \mathrm{sec}$ and extension at $72^{\circ} \mathrm{C}$ for $30 \mathrm{sec}$. The relative mRNA expression levels were calculated using the $2^{-\Delta \Delta C q}$ method (10) and normalized to the levels of the internal reference gene GAPDH, and the miRNA levels were normalized to U6. The primer sequences used were the following: LINC00963 forward, 5'-GGTAAATCGAGG CCCAGAGAT-3' and reverse, 5'-ACGTGGATGACAGCG TGTGA-3'; GAPDH forward, 5'-CACATCGCTCAGACA CCATG-3' and reverse, 5'-TGACGGTGCCATTGGAAT TT-3'; miR-27b forward, 5'-CCGGCCTTCACAGTGGCTA-3' and reverse, 5'-CGGGTCGGTGGCAGAACTT-3'; and U6 forward, 5'-CTCGCTTCGGCAGCACATATA-3' and reverse, 5'-ACGCTTCACGAATTTGAGTGTC-3'.

Cell transfection. Two short hairpin RNAs (shRNAs) against LINC00963 (shRNA-LINC00963-1 and shRNA-LINC00963-2; 500 ng/ $\mu$; Guangzhou RiboBio Co., Ltd.) were used for the specific knockdown of LINC00963. The shRNA clone containing non-sense shRNA sequences served as a negative control (shRNA-NC; $500 \mathrm{ng} / \mu \mathrm{l}$ ). In addition, two miR-27b inhibitors (miR-27b inhibitor-1 and miR-27b inhibitor-2; 100 pmol) and a corresponding negative control (miR-NC; 100 pmol) were purchased from Shanghai GenePharma Co., Ltd. All shRNAs and inhibitors were separately transfected into HRECs cells (density, $1 \times 10^{5}$ cells/well) using the Lipofectamine ${ }^{\circledR} 2000$ reagent (Invitrogen; Thermo Fisher Scientific, Inc.) at $37^{\circ} \mathrm{C}$. Subsequent assays were carried out at $24 \mathrm{~h}$ following transfection.

Western blotting. HRECs were harvested and total proteins were extracted using RIPA lysis buffer (Beyotime Institute of Biotechnology) supplemented with protease inhibitors (dilution, 1:100; Beyotime Institute of Biotechnology). The lysates were centrifuged at $850 \mathrm{x}$ g for $15 \mathrm{~min}$ at $4^{\circ} \mathrm{C}$. The supernatant was collected and mixed with a loading buffer (Beyotime Institute of Biotechnology) containing $100 \mathrm{mM}$ dithiothreitol. Western blot analysis was subsequently performed. Briefly, total proteins were quantified using a protein concentration determination kit (Beyotime Institute of Biotechnology), and proteins $(30 \mu \mathrm{g} /$ lane) were separated by $15 \%$ SDS-PAGE. The separated proteins were subsequently transferred onto PVDF membranes (EMD Millipore) and blocked with 5\% BSA (Beyotime Institute of Biotechnology) at room temperature for $2 \mathrm{~h}$. The membranes were then incubated with the following primary antibodies (all purchased from Abcam) at $4^{\circ} \mathrm{C}$ overnight: Anti-cyclin-dependent kinase 2 (anti-CDK2; cat. no. ab29; 1:1,000), anti-Ki67 (cat. no. ab15580; 1:1,000) and anti-GAPDH (cat. no. ab8245; 1:2,000). Following incubation with the primary antibody, the membranes were washed with TBST $(0.1 \%$ Tween-20) and incubated at room temperature for $1.5 \mathrm{~h}$ with a horseradish peroxidase-conjugated goat anti-rabbit IgG secondary antibody (1:5,000; cat. no. SA00001-9) or a goat anti-mouse IgG secondary antibody (1:5,000; cat. no. SA00001-8; both from ProteinTech Group, Inc.). Protein bands were visualized using a luminol 
A

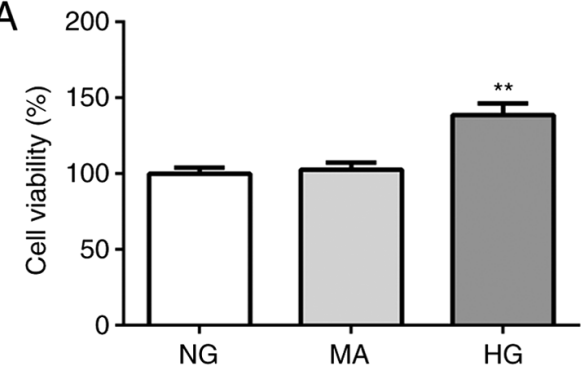

$\mathrm{C}$

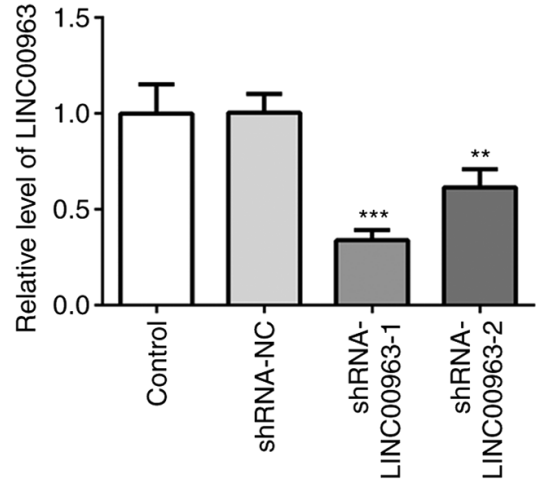

B
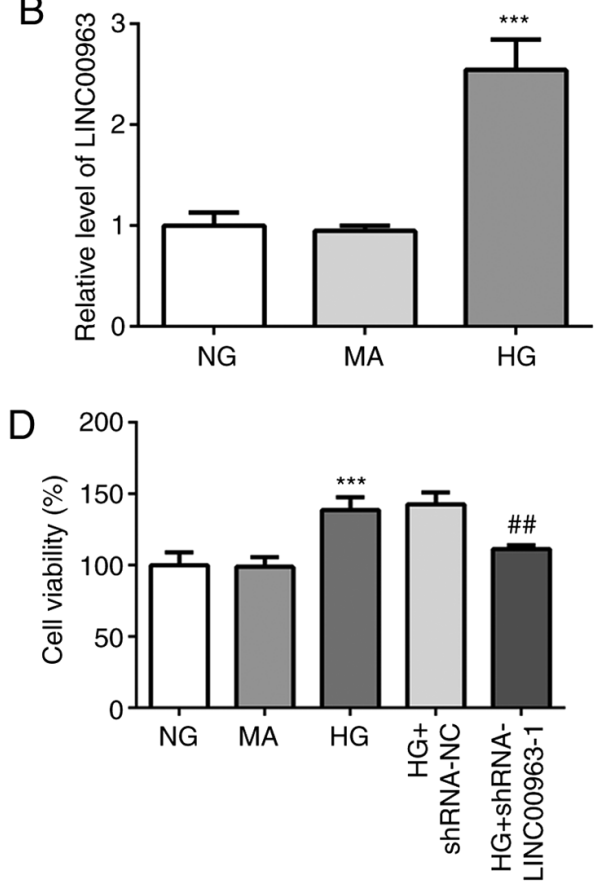
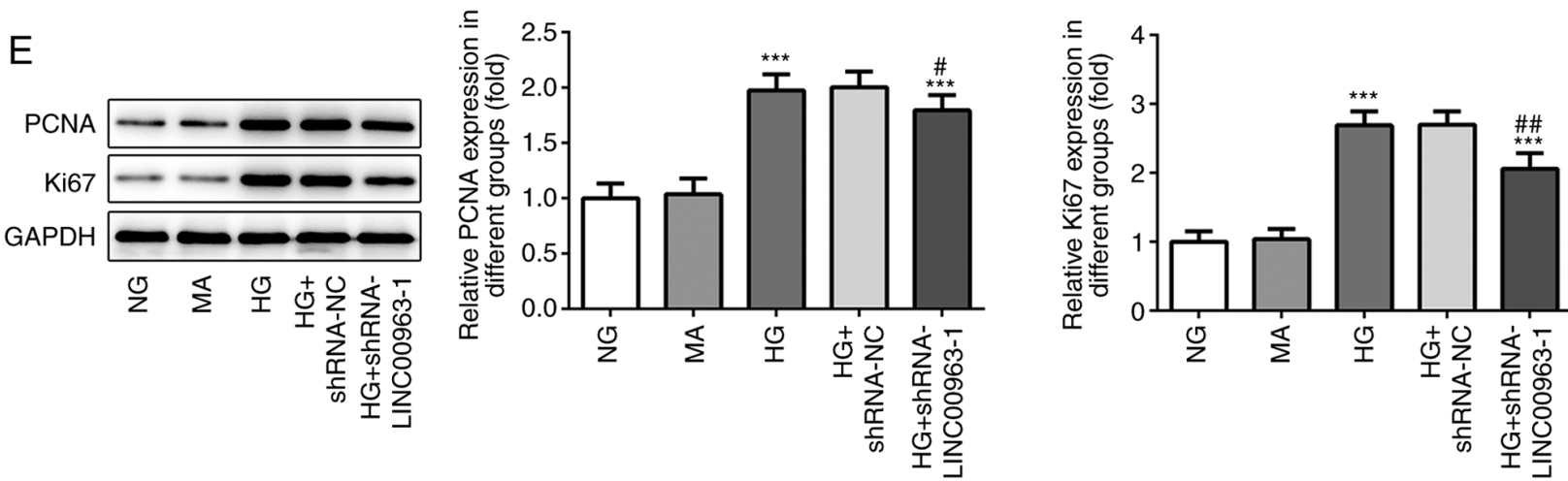

Figure 1. HREC viability and LINC00963 expression. (A) Viability of HRECs treated with different concentrations of glucose. (B) Expression levels of LINC00963 in HRECs treated with high and low concentrations of glucose and mannitol. (C) Expression levels of LINC00963 in different groups following LINC00963 knockdown. (D) HREC viability was notably attenuated following LINC00963 knockdown. (E) Protein expression levels of PCNA and Ki67 with different concentrations of glucose and following LINC00963 knockdown. All experiments were repeated three times. ${ }^{* * *} \mathrm{P}<0.01$ and ${ }^{* * * *} \mathrm{P}<0.001$ vs. NG group; ${ }^{\#} \mathrm{P}<0.05$ and ${ }^{\# \#} \mathrm{P}<0.01$ vs. HG group. LINC00963, long intergenic non-protein-coding RNA 963; HRECs, human retinal capillary endothelial cells; PCNA, proliferating cell nuclear antigen; NG group, normal glucose group; HG group, high glucose group; MA, mannitol group; shRNA, short hairpin RNA; NC, negative control.

reagent (Santa Cruz Biotechnology, Inc.) and analyzed using ImageJ software (version 1.48; National Institutes of Health).

Wound healing assay. HRECs were seeded into a 6-well plate (density, $6 \times 10^{4}$ cells/well) and $24 \mathrm{~h}$ following treatment with different concentrations of glucose, cultured until 80-90\% and subjected to serum-free starvation for $4 \mathrm{~h}$ (Gibco; Thermo Fisher Scientific, Inc.). Subsequently, a wound across the confluent cell monolayer was created in the cell monolayer using a 200- $\mu 1$ pipette tip, and the cells were then washed with culture medium to remove the non-adherent cells. Images were then captured under a light microscope (Olympus Corporation) at $0 \mathrm{~h}$ and after incubation with serum-free medium for $24 \mathrm{~h}$ at $37^{\circ} \mathrm{C}$ in a humified atmosphere of $5 \% \mathrm{CO}_{2}$ (magnification, $\mathrm{x} 100$ ). Finally, an image measurement software (Digimizer, v4.2.6; MedCalc Software bvba) was utilized to calculate the relative migration distance of cells in each group according to the following formula: Migration distance=scratch width at $0 \mathrm{~h}$-scratch width at $24 \mathrm{~h}$.

Transwell invasion assay. A Transwell invasion assay was performed to measure the cell invasion ability. Briefly, $2 \times 10^{5}$ HRECs were resuspended in $100 \mu 1$ serum-free medium (Thermo Fisher Scientific, Inc.) and plated into the upper chambers of a 24 -well Transwell plate $(8-\mu \mathrm{m}$ pore size; Corning, Inc.) precoated with Matrigel ${ }^{\mathrm{TM}}$ (BD Biosciences) for $24 \mathrm{~h}$ at $37^{\circ} \mathrm{C}$ following transfection. RPMI-1640 medium supplemented with $20 \%$ FBS was added to the lower chamber to act as a chemoattractant. Following $24 \mathrm{~h}$ of incubation at $37^{\circ} \mathrm{C}$, the invading HRECs on the bottom surface of the filter were fixed with $100 \%$ methanol at $4^{\circ} \mathrm{C}$ for $30 \mathrm{~min}$ and stained with hematoxylin at room temperature for $20 \mathrm{~min}$. Cell invasion was evaluated in three randomly selected fields under a fluorescence microscope (magnification, x20). 


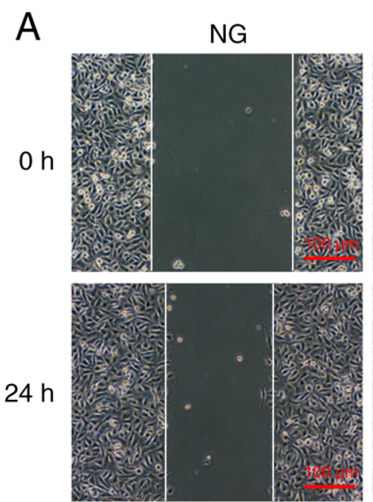

B

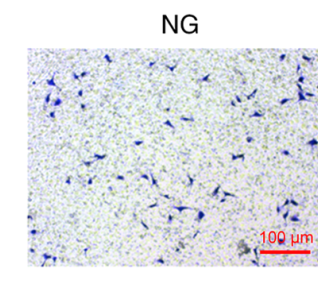

C

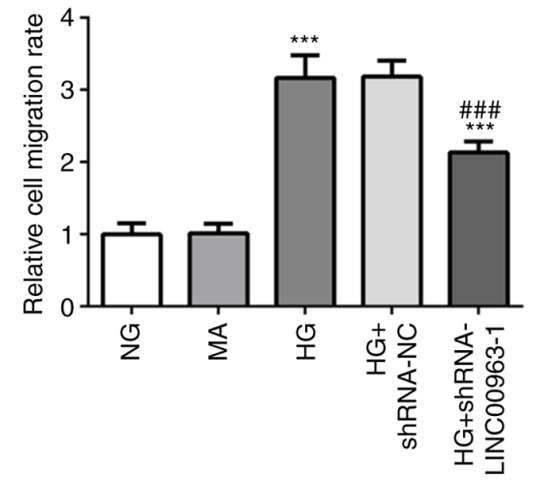

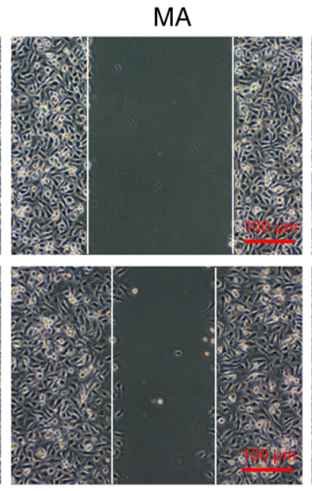

MA
$H G$

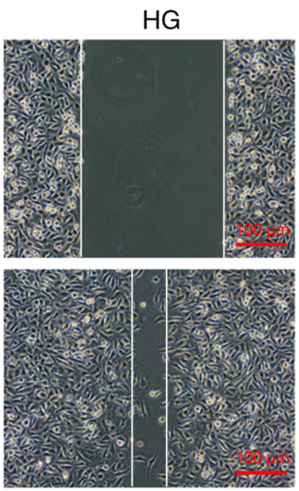

HG
$H G+$ shRNA-NC

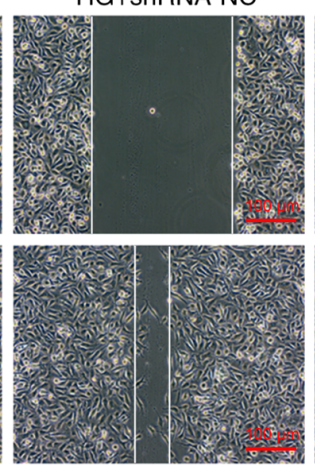

HG+shRNA-NC
HG+shRNA-LINC00963-1
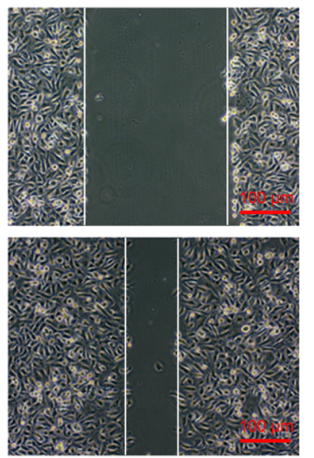

HG+ShRNA-LINC00963-1
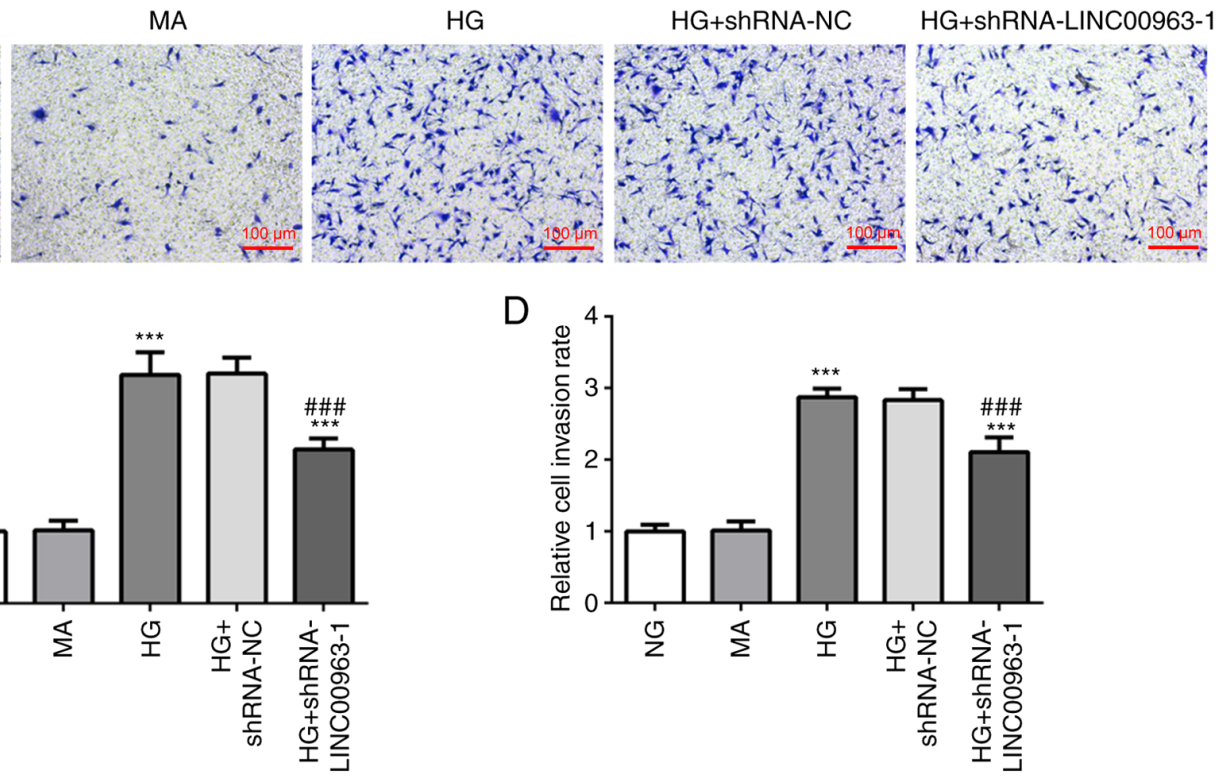

Figure 2. LINC00963 knockdown attenuates HREC migration and invasion. (A) Effects of LINC00963 knockdown on the migration ability of HESCs. (B) Effects of LINC00963 knockdown on the invasion ability of HESCs. Relative cell (C) migration and (D) invasion rates. All the experiments were repeated three times. ${ }^{* * *} \mathrm{P}<0.001$ vs. NG group; ${ }^{\# \# \#} \mathrm{P}<0.001$ vs. HG group. LINC00963, long intergenic non-protein-coding RNA 963; HRECs, human retinal capillary endothelial cells; NG group, normal glucose group; HG group, high glucose group; MA, mannitol group; shRNA, short hairpin RNA; NC, negative control.

Statistical analysis. All experiments were independently repeated three times. Statistical analyses were performed using the GraphPad Prism 5 software (GraphPad Software, Inc.). All data are expressed as the mean \pm SEM, unless otherwise specified. The statistical significance of the differences between two groups were evaluated using an unpaired two-tailed Student's t-test, while one-way ANOVA followed by Tukey's post hoc test was performed to analyze differences among multiple groups. $\mathrm{P}<0.05$ was considered to indicate a statistically significant difference.

\section{Results}

HREC viability and LINC00963 expression in cells treated with different concentrations of glucose. As shown in Fig. 1A, cell viability was significantly higher in the HG group compared with that in the NG group. The expression levels of LINC00963 were evaluated in HRECs treated with high and low concentrations of glucose and mannitol (Fig. 1B). Compared with the NG group, the expression levels of LINC00963 were found to be significantly higher in the HG group.
Effect of LINC00963 knockdown on HRECs. As shown in Fig. 1C, transfection of HRECs with shRNA-LINC00963-1 silenced LINC00963 expression more efficiently compared with shRNA-LINC00963-2 (0.33 vs. 0.62, respectively). Therefore, shRNA-LINC00963-1 was utilized for subsequent experiments. The cell viability in the HG + shRNA-LINC00963-1 group was notably attenuated compared with the HG group (Fig. 1D). Furthermore, the protein expression levels of proliferating cell nuclear antigen (PCNA) and Ki67 were no significantly different between the NG and MA groups (Fig. 1E). In addition, the expression of PCNA and Ki67 was upregulated in the HG group compared with the NG and MA groups, and it was downregulated in the HG + shRNA-LINC00963-1 group compared with the HG group (Fig. 1E).

LINC00963 knockdown attenuates the migration and invasion ability of HRECs. Following incubation for $24 \mathrm{~h}$, the scratches in the cell monolayer were wider in the NG and MA groups compared with those in the HG group (Fig. 2A), indicating that the cell migration ability was enhanced in a high-glucose environment. Furthermore, compared with the HG group, the scratches were wider in the HG + shRNA-LINC00963-1 group, 
A

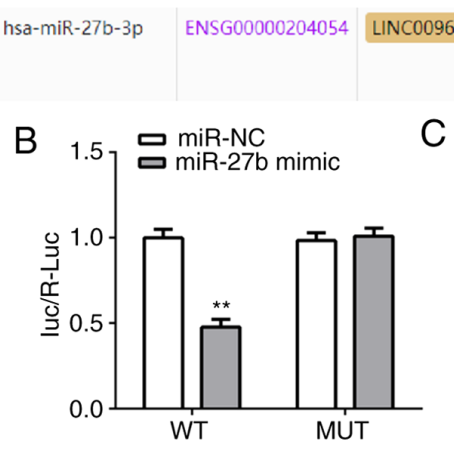

processed_transcript chr9:132264496-132264517[+]

Target: 5 ' guGGGGCUGUGUUGACUGUGAg $3^{\prime}$ miRna : 3' agUCUUGA-AUCGGUGACACUU 5
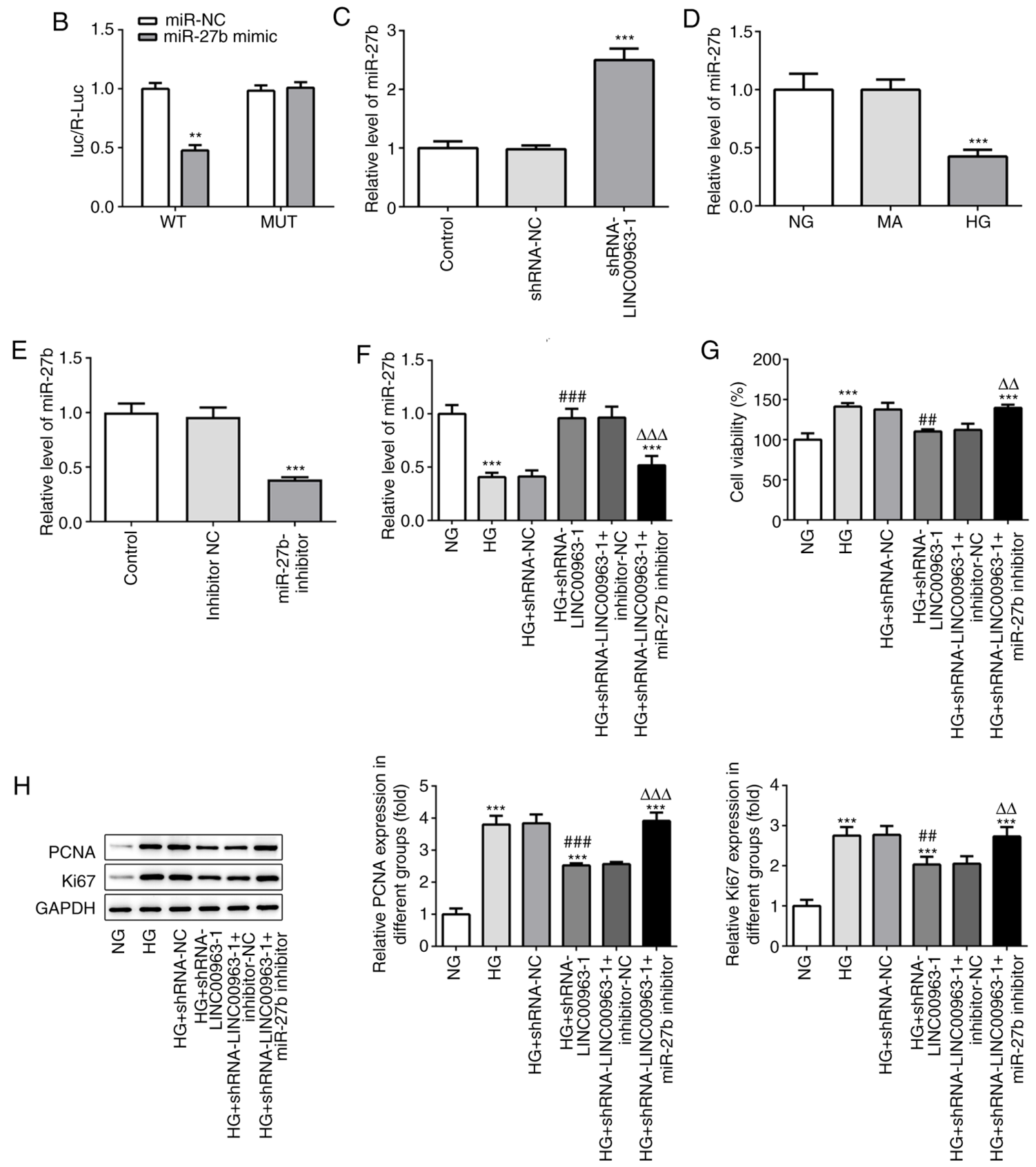

Figure 3. LINC00963 directly targets miR-27b. (A) Complementary binding site between the 3'-untranslated region of LINC00963 and miR-27b was predicted using the StarBase database. (B) Dual-luciferase reporter assay was performed to evaluate the association between LINC00963 and miR-27b. ${ }^{* *} \mathrm{P}<0.01 \mathrm{vs}$. miR-NC. (C) RT-qPCR was used to determine the relative expression levels of miR-27b in HRECs following LINC00963 knockdown. (D) RT-qPCR was used to determine the relative expression levels of miR-27b in HRECs treated with different concentrations of glucose. (E) RT-qPCR analysis revealed that the miR-27b expression levels were downregulated in HRECs following transfection with miR-27b inhibitor. (F) RT-qPCR was used to determine the relative expression levels of miR-27b following HG, HG + shRNA-NC, HG + shRNA-LINC00963-1, HG + shRNA-LINC00963-1 + inhibitor-NC and HG + shRNA-LINC00963-1 + miR-27b inhibitor. (G) HREC viability following transfection with miR-27b inhibitor. (H) Protein expression levels of PCNA and Ki67. All experiments were repeated three times. ${ }^{* * *} \mathrm{P}<0.001$ vs. NG group; ${ }^{\# \#} \mathrm{P}<0.01,{ }^{, \# \#} \mathrm{P}<0.001$ vs. HG group; ${ }^{\Delta \Delta} \mathrm{P}<0.01,{ }^{\Delta \Delta \Delta} \mathrm{P}<0.001$ vs. HG + shRNA-LINC00963-1 group. LINC00963, long intergenic non-protein-coding RNA 963; HRECs, human retinal capillary endothelial cells; PCNA, proliferating cell nuclear antigen; NG group, normal glucose group; HG group, high glucose group; MA, mannitol group; RT-qPCR, reverse transcription-quantitative PCR; miR, microRNA; shRNA, short hairpin RNA; NC, negative control.

suggesting that LINC00963 knockdown could attenuate the migration and invasion ability of HRECs. It was also shown that transfection with shRNA-LINC00963-1 decreased the migration ability of HRECs (Fig. 2B).
LINC00963 directly targets miR-27b. To reveal the molecular mechanisms underlying the role of LINC00963 in HRECs, the ENCORI database (starBase v2.0, http://starbase.sysu. edu.cn) was utilized. The analysis predicted that LINC00963 
A

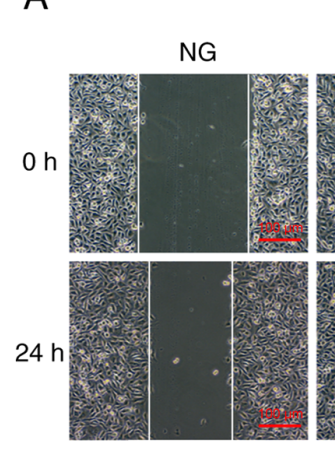

B

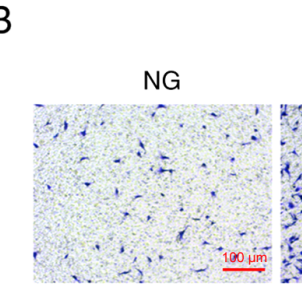

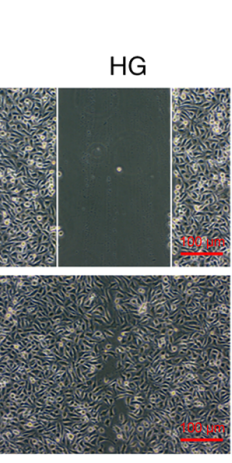

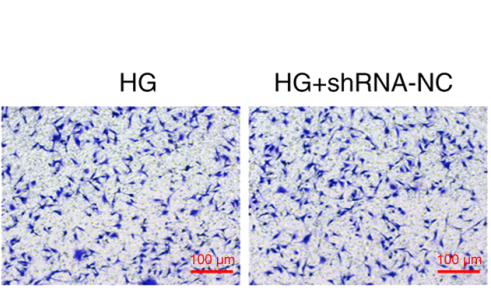

$\mathrm{C}$

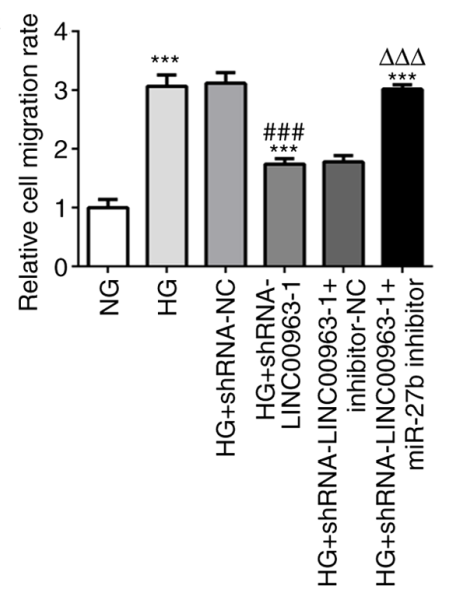

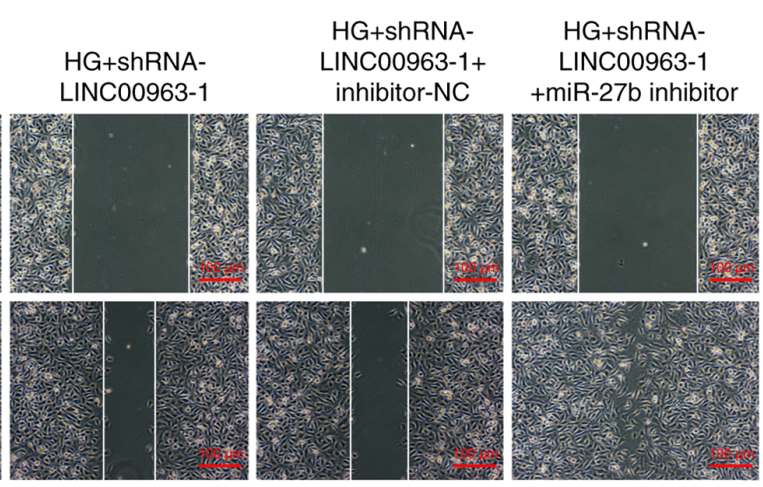

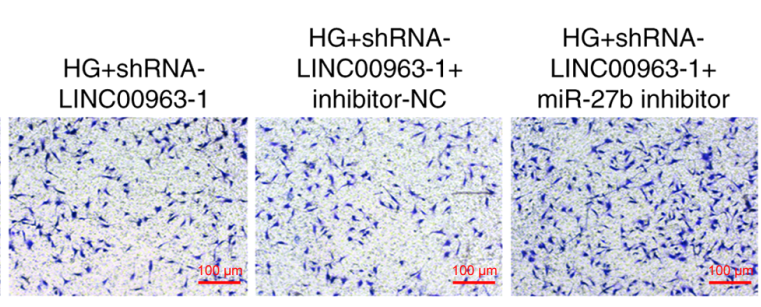

$\mathrm{D}$

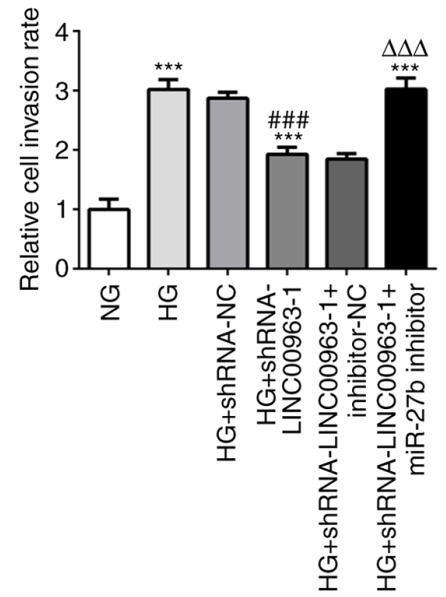

Figure 4. miR-27b silencing can restore the anti-migratory and anti-invasive effects of LINC00963 knockdown. (A) Cell migration rate was measured in HRECs co-transfected with shRNA-LINC00963-1 with or without miR-27b inhibitor using a wound healing assay (magnification, x100). (B) Cell invasion rate was determined in HERCs co-transfected with shRNA-LINC00963-1 with or without miR-27b inhibitor using a Transwell assay (magnification, x100).

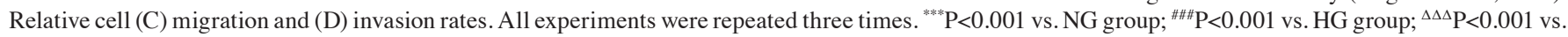
HG + shRNA-LINC00963-1 group. LINC00963, long intergenic non-protein-coding RNA 963; HRECs, human retinal capillary endothelial cells; NG group, normal glucose group; HG group, high glucose group; miR, microRNA; shRNA, short hairpin RNA; NC, negative control.

could directly target miR-27b (Fig. 3A). miR-27b has been reported to be a tumor suppressor gene. miR-27b can inhibit epithelial-mesenchymal transition andinhibit the proliferation and migration of oral squamous cell carcinoma by targeting integrin subunit $\alpha 5$ (11). In addition, miR-27b inhibited the growth and metastatic behavior of ovarian cancer cells by targeting C-X-C motif chemokine ligand 1 (12). In the present study, the association between LINC00963 and miR-27b was confirmed by dual-luciferase reporter assays, demonstrating that co-transfection of HRECs with WT LINC00963-Luc and miR-27b mimics significantly reduced the relative luciferase activity of LINC00963-Luc. In addition, cell viability was enhanced in the HG + shRNA-LINC00963-1 + miR-27b inhibitor group compared with the $\mathrm{HG}+$ shRNA-LINC00963-1 group. Additionally, the expression levels of PCNA and Ki67 were upregulated in the $\mathrm{HG}+$ shRNA-LINC00963-1 + miR-27b inhibitor group compared with those in the HG + shRNA-LINC00963-1 group. The aforementioned findings indicated that miR-27b inhibited the proliferation of HRECs (Fig. 3B-H). As shown in Fig. 4, miR-27b attenuated the wound healing and migration rate of HRECs.

\section{Discussion}

Diabetes, which is caused by the abnormal secretion and/or inability of the body to use insulin, is one of the most common non-communicable diseases in clinical practice $(13,14)$. In 2017, 425 million individuals, aged 20-79 years, were diagnosed with diabetes worldwide. This number is estimated to increase to 629 million by 2045 (15). Clinically, diabetes progresses slowly over several years and is accompanied by several complications. Targeted nursing, including proper control of patients' blood glucose and real-time monitoring of physical data, may be effective in improving the cure rate of patients with diabetes $(16,17)$. DR is one of the most common 
complications of diabetes. Hyperglycemia can damage the retinal vascular system and endothelial cells and thicken the basement membrane $(18,19)$. In the present study, the viability of HRECs in a high-glucose environment was determined by a CCK- 8 assay. It was demonstrated that cell viability was higher in the HG group compared with the NG group, suggesting that the high-glucose environment may enhance the viability of HRECs.

LINC00963 is non-coding RNA (20) that is considered to act as a regulatory factor of cell apoptosis (21). LINC00963 has recently been reported to promote breast and hepatocellular carcinogenesis $(22,23)$. Its expression is upregulated in several types of cancer, such as melanoma and bladder cancer $(24,25)$. However, the association between LINC00963 and diabetes has not been reported to date. Thus, the expression of LINC00963 was measured by RT-qPCR in the present study, and the results demonstrated that its expression was upregulated under high-glucose conditions. It has been reported that PCNA, an essential protein for nuclear DNA synthesis, is widely involved in cell proliferation and invasion $(26,27)$. In addition, Ki67, a nuclear antigen protein involved in cell proliferation (28), is closely associated with cell mitosis, and its expression reflects the normal proliferative activity of cells (29). Western blot analysis revealed that, compared with the NG group, the expression levels of PCNA and Ki67 were elevated in the HG group, suggesting that cells in the HG group exhibited strong proliferation ability. However, downregulation of PCNA and Ki67, mediated by LINC00963 silencing, indicated that LINC00963 downregulation may inhibit cell proliferation. Next, migration and invasion experiments were performed, and the results demonstrated that treatment of HRECs with shRNA-LINC00963-1 attenuated cell migration, indicating that LINC00963 may promote cell migration.

Subsequently, bioinformatics analysis using the ENCORI database predicted that miR-27b could directly interact with the LINC00963 3'-UTR. miR-27 has been proven to play contradictory roles in different diseases. miR-27b was reported to act as a tumor suppressor in gastric cancer, exhibiting reduced expression in human gastric cancer tissues and cells (30). It has been demonstrated that miR-27b promotes fibroblast-like synovial cell apoptosis (18), as well as endothelial and glioma cell proliferation (31). In the present study, the cell proliferation and migration rates, and the protein expression levels of PCNA and Ki67 were increased in the miR-27b inhibitor group, suggesting that miR-27b may attenuate cell proliferation. Furthermore, miR-27b inhibitor reversed the inhibitory effect of LINC00963 on cell migration in response to high-glucose stimulation.

In summary, LINC00963 may regulate the proliferation and apoptosis of HERCs via targeting miR-27b. The findings of the present study may contribute to the clinical diagnosis of DR and provide a theoretical basis for research into effective drugs for the treatment of this condition. However, there were certain limitations to the present study. First, no in vivo experiments were performed. Second, the molecular mechanism underlying the effects of LINC00963 silencing on miR-27b expression and HREC function was not fully investigated Therefore, these issues require further in-depth research and will be addressed in future studies.

\section{Acknowledgements}

Not applicable.

\section{Funding}

No funding was received.

\section{Availability of data and materials}

The datasets used and/or analyzed during the current study are available from the corresponding author on reasonable request.

\section{Authors' contributions}

RZ and CN designed the study and wrote the manuscript; YG performed the experiments; JW collected and analyzed the data; LH interpreted the data. RZ and LH can confirm the authenticity of the raw data. All the authors have read and approved the final manuscript. All authors confirm the authenticity of the raw data.

\section{Ethics approval and consent to participate}

Not applicable.

\section{Patient consent for publication}

Not applicable.

\section{Competing interests}

The authors declare that they have no competing interests.

\section{References}

1. Dall TM, Yang W, Halder P, Pang B, Massoudi M, Wintfeld N, Semilla AP, Franz J and Hogan PF: The economic burden of elevated blood glucose levels in 2012: Diagnosed and undiagnosed diabetes, gestational diabetes mellitus, and prediabetes. Diabetes Care 37: 3172-3179, 2014.

2. Patterson CC, Karuranga S, Salpea P, Saeedi P, Dahlquist G, Soltesz G and Ogle GD: Worldwide estimates of incidence, prevalence and mortality of type 1 diabetes in children and adolescents: Results from the International diabetes federation diabetes atlas, 9th edition. Diabetes Res Clin Pract 157: 107842, 2019.

3. Yau JW, Rogers SL, Kawasaki R, Lamoureux EL, Kowalski JW, Bek T, Chen SJ, Dekker JM, Fletcher A, Grauslund J, et al: Global prevalence and major risk factors of diabetic retinopathy. Diabetes Care 35: 556-564, 2012.

4. Savage SR, McCollum GW, Yang R and Penn JS: RNA-seq identifies a role for the PPAR $\beta / \delta$ inverse agonist GSK0660 in the regulation of $\mathrm{TNF} \alpha$-induced cytokine signaling in retinal endothelial cells. Mol Vis 21: 568-576, 2015.

5. Quinn JJ and Chang HY: Unique features of long non-coding RNA biogenesis and function. Nat Rev Genet 17: 47-62, 2016.

6. Pan C, Wang X, Shi K, Zheng Y, Li J, Chen Y, Jin L and Pan Z: MiR-122 reverses the doxorubicin-resistance in hepatocellular carcinoma cells through regulating the tumor metabolism. PLoS One 11: e0152090, 2016.

7. Reichholf B, Herzog VA, Fasching N, Manzenreither RA, Sowemimo I and Ameres SL: Time-resolved small RNA sequencing unravels the molecular principles of microRNA homeostasis. Mol Cell 75: 756-768.e7, 2019.

8. Ruiz MA, Feng B and Chakrabarti S: Polycomb repressive complex 2 regulates MiR-200b in retinal endothelial cells: Potential relevance in diabetic retinopathy. PLoS One 10: e0123987, 2015. 
9. Ding L, Ni J, Yang F, Huang L, Deng H, Wu Y, Ding X and Tang J: Promising therapeutic role of miR-27b in tumor. Tumour Biol 39: 1010428317691657, 2017.

10. Livak KJ and Schmittgen TD: Analysis of relative gene expression data using real-time quantitative PCR and the 2(-Delta Delta C(T)) method. Methods 25: 402-408, 2001.

11. Li T, Wu Q, Liu D and Wang X: MiR-27b Suppresses tongue squamous cell carcinoma epithelial-mesenchymal transition by targeting ITGA5. Onco Targets Ther 13: 11855-11867, 2020.

12. Liu CH, Jing XN, Liu XL, Qin SY, Liu MW and Hou CH: Tumor-suppressor miRNA-27b-5p regulates the growth and metastatic behaviors of ovarian carcinoma cells by targeting CXCL1. J Ovarian Res 13: 92, 2020.

13. Murai N, Saiki R, Hashizume M, Kigawa Y, Koizumi G, Tadokoro R, Endo K, Iizaka T, Otsuka F, Izumizaki M and Nagasaka S: Alcohol flushing is independently associated with lesser degree of carotid atherosclerosis in Japanese type 2 diabetic patients. Diabetol Int 9: 68-74, 2017.

14. Chong PL, Pisharam J, Abdullah A and Chong VH: Gestational diabetes insipidus. QJM 112: 123-124, 2019.

15. Wong TY and Sabanayagam C: The war on diabetic retinopathy: Where are we now? Asia Pac J Ophthalmol (Phila) 8: 448-456, 2019.

16. Wilson M, Chen HS and Wood M: Impact of nurse champion on quality of care and outcomes in type 2 diabetes patients. Int J Evid Based Healthc 17: 3-13, 2019.

17. Allen D: The nurse's role in childhood diabetes. Nurs Child Young People 28: 11, 2016.

18. Mohammad HMF, Sami MM, Makary S, Toraih EA, Mohamed AO and El-Ghaiesh SH: Neuroprotective effect of levetiracetam in mouse diabetic retinopathy: Effect on glucose transporter-1 and GAP43 expression. Life Sci 232: 116588, 2019

19. Clore JN and Thurby-Hay L: Glucocorticoid-induced hyperglycemia. Endocr Pract 15: 469-474, 2009.

20. Xie LB, Chen B, Liao X, Chen YF, Yang R, He SR, Pei LJ and Jiang R: LINC00963 targeting miR-128-3p promotes acute kidney injury process by activating JAK2/STAT1 pathway. J Cel Mol Med 24: 5555-5564, 2020

21. Wang L, Han S, Jin G, Zhou X, Li M, Ying X, Wang L, Wu H and Zhu Q: Linc00963: A novel, long non-coding RNA involved in the transition of prostate cancer from androgen-dependence to androgen-independence. Int J Oncol 44: 2041-2049, 2014.
22. Zhang N, Zeng X, Sun C, Guo H, Wang T, Wei L, Zhang Y, Zhao J and Ma X: LncRNA LINC00963 promotes tumorigenesis and radioresistance in breast cancer by sponging miR-324-3p and inducing ACK1 expression. Mol Ther Nucleic Acids 18: 871-881, 2019.

23. Wu JH, Tian XY, An QM, Guan XY and Hao CY: LINC00963 promotes hepatocellular carcinoma progression by activating PI3K/AKT pathway. Eur Rev Med Pharmacol Sci 22: 1645-1652, 2018.

24. Jiao H, Jiang S, Wang H, Li Y and Zhang W: Upregulation of LINC00963 facilitates melanoma progression through miR-608/NACC1 pathway and predicts poor prognosis. Biochem Biophys Res Commun 504: 34-39, 2018.

25. Zhou N, Zhu X and Man L: LINC00963 functions as an oncogene in bladder cancer by regulating the miR-766-3p/MTA1 axis Cancer Manag Res 12: 3353-3361, 2020.

26. Chatzileontiadou DSM, Samiotaki M, Alexopoulou AN, Cotsiki M, Panayotou G, Stamatiadi M, Balatsos NAA, Leonidas DD and Kontou M: Proteomic analysis of human angiogenin interactions reveals cytoplasmic PCNA as a putative binding partner. J Proteome Res 16: 3606-3622, 2017.

27. Wang X, Wang D, Yuan N, Liu F, Wang F, Wang B and Zhou D: The prognostic value of PCNA expression in patients with osteosarcoma: A meta-analysis of 16 studies. Medicine (Baltimore) 96: e8254, 2017.

28. Rahmanzadeh R, Huttmann G, Gerdes J and Scholzen T: Chromophore-assisted light inactivation of pKi-67 leads to inhibition of ribosomal RNA synthesis. Cell Prolif 40: 422-430, 2007.

29. Scholzen T and Gerdes J: The Ki-67 protein: From the known and the unknown. J Cell Physiol 182: 311-322, 2000.

30. Feng Q, Wu X, Li F, Ning B, Lu X, Zhang Y, Pan Y and Guan W: MiR-27b inhibits gastric cancer metastasis by targeting NR2F2. Protein Cell 8: 114-122, 2017.

31. Urbich C, Kaluza D, Fromel T, Knau A, Bennewitz K, Boon RA, Bonauer A, Doebele C, Boeckel JN, Hergenreider E, et al: MicroRNA-27a/b controls endothelial cell repulsion and angiogenesis by targeting semaphorin 6A. Blood 119: 1607-1616, 2012.

c) (i) $\odot$ This work is licensed under a Creative Commons EY NC NO Attribution-NonCommercial-NoDerivatives 4.0 International (CC BY-NC-ND 4.0) License. 